\title{
0084 HELMETS FOR SNOW SPORTS IN AUSTRALIA: PREVALENCE, PREDICTORS, RECENT TRENDS AND ATTITUDES OF USE
}

T P Cundy,* B J Systermans, W J Cundy, P J Cundy, N E Briggs, J B Robinson Correspondence: University of Adelaide, South Australia, Falls Creek Medical Centre, Victoria, Australia. Personal: 6 Torrens Street, College Park, South Australia 5069, Australia

\subsection{6/ip.2010.029215.84}

Background Traumatic head injury is the leading cause of mortality and serious morbidity in snow sports and is increasing in incidence. Helmet use in snow sports can reduce incidence of head injury by up to $60 \%$. Mandatory helmet use is not legislated in recreational settings for snow sports. This study aimed to quantify prevalence and trends of helmet use by skiers and snowboarders as well as investigate predictors of helmet use and reasons why they are worn.

Methods A cross-sectional study including observation of skiers and snowboarders allocated to adult and child groups (5267), retrospective review of ski patrol accident report forms recorded between 2003 and 2008 (3984) and completion of a specially designed questionnaire by randomly approached snow sport participants (1029).

Results In $2008,16 \%$ of adults and $67 \%$ of children wore helmets. Helmet use increased for adults and children between 2003 and 2008. Children, males and snowboarders were significantly more likely to wear a helmet than respective counterparts. Significant predictors of helmet use were level of experience, past major crash requiring medical assistance, snow sport lessons and location of activity. Key reasons for helmet use and disuse were identified.

Conclusions Child helmet use far exceeded adult helmet use. Rates of voluntary use are increasing but many remain unprotected from benefits of a helmet. Identification of those least likely to wear a helmet and their reasons for not doing so should allow targeted helmet promotion and injury prevention strategies to reduce serious head injuries in snow sports. 